\section{Bases fisiopatológicas para una clasificación de la neuropatía diabética}

\author{
PABLO R. OLMOS ${ }^{1,2}$, SERGIO NIKLITSCHEK, \\ ROBERTO I. OLMOS ${ }^{\mathrm{a}}$, JORGE I. FAÚNDEZ ${ }^{\mathrm{a}}$, \\ THOMAS A. QUEZADA, MILAN A. BOZINOVIC ${ }^{a}$, \\ IAN A. NIKLITSCHEK ${ }^{\text {a }}$, JORGE ACOSTA ${ }^{\text {a }}$, \\ CLAUDIO N. VALENCIA ${ }^{1}$, FELIPE A. BRAVO
}

\section{A new physiopathological classification of diabetic neuropathy}

\begin{abstract}
Nowadays, Diabetic Neuropathy $(D N)$ is considered the most common cause of peripheral neuropathy in clinical practice. It can affect sensitive, motor or autonomic nerve fibers, with symmetric, asymmetric, acute or chronic presentations. Due to this variability, with multiple physiopathologic mechanisms involved, a complex clinical classification has been used until recently. The aim of this review is to present a new classification of diabetic neuropathy, based on its physiopathology. It is divided in metabolic microvascular and hypoxic, autoimmune and inflammatory, compressive, secondary to complications of diabetes and related to treatment. It must be understood that DN is not just a functional disease, but a complication of diabetes with molecular and pathological substrates caused by hyperglycemia. Therefore, normalization of blood glucose is a fundamental step towards the successful prevention and treatment
\end{abstract} of DN.

(Rev Med Chile 2012; 140: 1593-1605).

Key words: Diabetes complications; Diabetic neuropathies; Physiopathology.

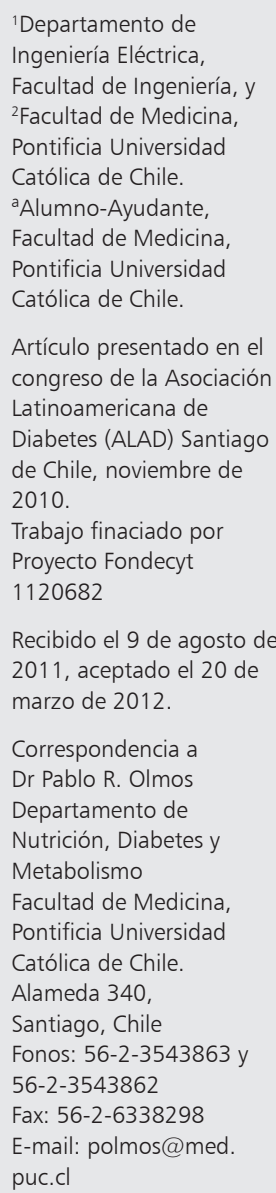

Recibido el 9 de agosto de 2011, aceptado el 20 de marzo de 2012.

Correspondencia a Dr Pablo R. Olmos Departamento de Nutrición, Diabetes y Metabolismo

Facultad de Medicina, Pontificia Universidad Católica de Chile.

Alameda 340,

Santiago, Chile Fonos: 56-2-3543863 y 56-2-3543862 Fax: 56-2-6338298 E-mail: polmos@med puc.cl

L a diabetes mellitus (DM) afecta a 243 millones de personas en el mundo, de los cuales 30 millones sufren de alguna forma de neuropatía diabética $(\mathrm{ND})^{1}$, que es la complicación más frecuente tanto de la diabetes mellitus tipo 1 (DM1) como de la diabetes mellitus tipo 2 (DM2) ${ }^{2}$. La ND se conoce desde el siglo V DC, cuando el médico Hindú Sustruta describió lo que hoy llamaríamos neuropatía dolorosa. Aunque en 1848 Claude Bernard aseguró que la diabetes mellitus era una enfermedad neurológica con manifestaciones metabólicas secundarias, su contemporáneo Marchal de Calvi aclaró que la ND era una consecuencia y no la causa de la diabetes mellitus ${ }^{3}$.

La primera clasificación de ND fue propuesta en el año $1893^{4}$, y actualmente la ND es la neuropatía más frecuente en clínica ${ }^{5}$, afectando hasta $50 \%$ de los pacientes al cabo de 25 años de evolución ${ }^{2}$.
Como medida del impacto de la ND en la calidad de vida de los diabéticos, basta tomar sus dos manifestaciones más frecuentes (ambas a las extremidades inferiores)-la pérdida de sensibilidad y la neuropatía dolorosa. La pérdida de sensibilidad protectora causa [a] úlceras de los pies (que afectan al 15\% de los diabéticos en algún momento de su vida $)^{6,7}, y$ [b] dos tercios de las amputaciones de extremidades inferiores en DM2. La neuropatía dolorosa afecta al 32,1\% de los DM2 y al 11,6\% de los DM1 en algún momento de sus vidas ${ }^{8}$.

Como la ND puede ir desde una leve disminución de la sensibilidad cutánea, hasta el dolor y la debilidad muscular extremas ${ }^{4}$, tradicionalmente se ha usado una clasificación sindromática ${ }^{9}$, que contiene 24 síndromes, de los cuales 16 son clínicos y 8 son subclínicos. Ya en el año 2005, en el contexto de avances en el conocimiento de la ND, 
se habían establecido las bases de una clasificación fisiopatológica de esta condición ${ }^{10}$.

En esta revisión, discutiremos los mecanismos patogénicos de la neuropatía diabética, en sus aspectos metabólicos, moleculares, vasculares e inflamatorios, para luego hacer un paralelo entre la más reciente clasificación clínica de neuropatía diabética, con los mecanismos fisiopatológicos involucrados.

Hasta hace 5 años, la fisiopatología de la ND podía resumirse en cinco aspectos ${ }^{11}$, a saber, [a] microangiopatía (daño de capilares y arteriolas) de los vasa nervorum, combinado con [b] edema axonal, [c] deterioro del transporte axonal, y [d] destrucción de la vaina de mielina. El resultado final sería [e] la pérdida axonal.

Este enfoque tradicional, sin embargo, es demasiado general, y no explica la gran variabilidad de presentaciones clínicas de la neuropatía diabética, con sus aspectos sensitivos, motores y autonómicos.

La Figura 1-A muestra un corte histológico de un nervio periférico mielinizado normal. La Figura 1-B muestra uno afectado por neuropatía diabética. Algunos axones se ven normales, otros han sido destruidos, y algunos muestran señales de regeneración de la vaina de mielina. Además, el vaso capilar del endoneuro muestra reducción de su diámetro, y su membrana basal muestra múltiples capas ${ }^{12}$.

\section{Fisiopatología-enfoque actual}

\section{Aspectos metabólicos}

Estos (Figura 2) incluyen (a) daño oxidante, (b) daño osmótico, y (c) inflamación.

Con la hiperglicemia, la glucosa que entra al axón y a la célula de Schwann, bajo la acción de la aldosa-reductasa se transforma en sorbitol, produciendo hiperosmolaridad y edema. Además, aumenta el NADP+ en desmedro del NADPH, paralizando la regeneración del glutatión, impidiendo la neutralización de los radicales oxidantes (ROS) y nitrosilantes (NOS), produciendo daño oxidante. Por otro lado, la mayor oferta de NADH desvía el metabolismo glucídico hacia diacilglicerol (DAG), activador de la proteína-kinasa-C (PKC), la que a su vez induce la producción de los mediadores TGF- $\beta 1$ (transforming-growth factor beta-uno) y NF- $\kappa \beta$ (Nuclear Factor Kappa beta) ${ }^{11}$, estimulantes ambos de la inflamación y la fibrosis.

\section{Aspectos vasculares}

Tradicionalmente se ha dicho que la microangiopatía intervendría en la génesis de la ND. Sin embargo, debemos enfatizar dos aspectos poco
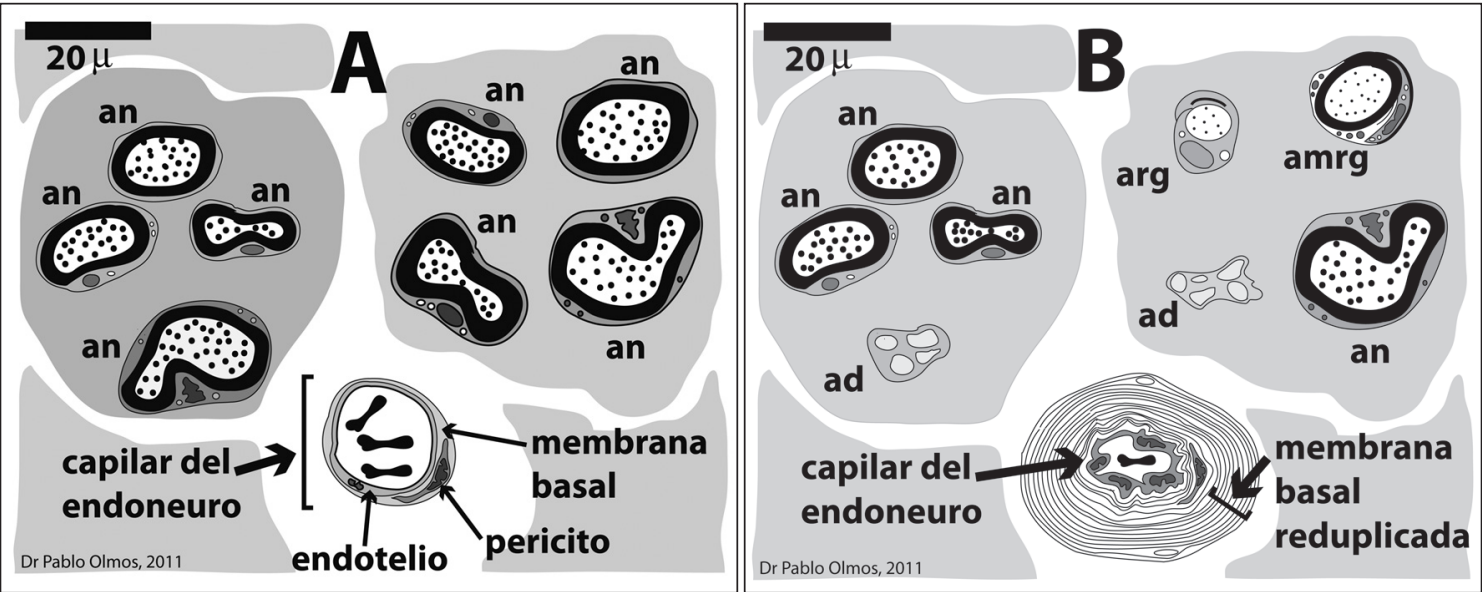

Figura 1. Histología de un nervio periférico con fibras mielinizadas. (A) Histología normal. Cada axón normal [an] está rodeada de una vaina de mielina [color negro] y en su interior hay microtúbulos [cortados]. Se observa también un vaso capilar del endoneuro, con su endotelio, membrana basal y pericito. (B) El mismo nervio periférico afectado por neuropatía diabética. Coexisten axones normales [an], axones destruidos [ad], axones en proceso de regeneración inicial [arg] y mielina en proceso de regeneración [amrg]. Es posible observar también la reducción del diámetro del capilar del endoneuro, y la reduplicación (engrosamiento por superposición de láminas) de su membrana basal ${ }^{12}$. 


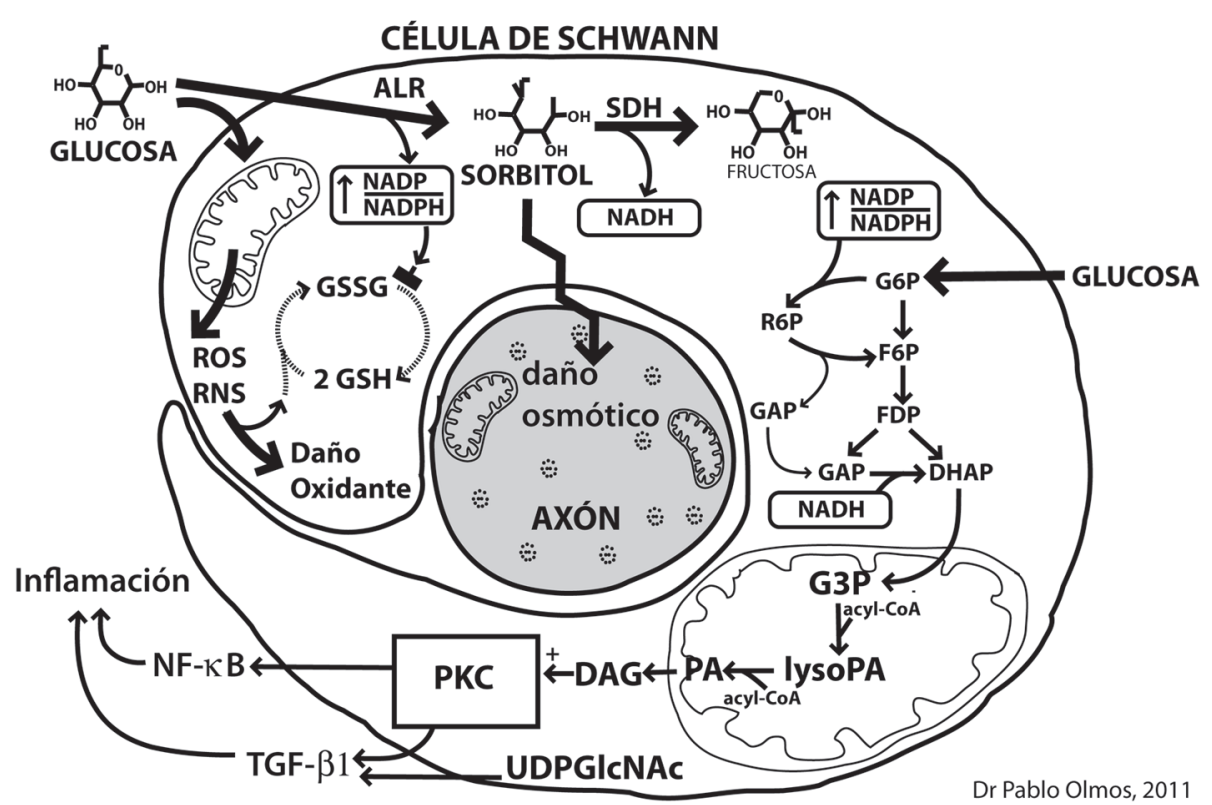

Figura 2. Los aspectos metabólicos de la fisiopatología de la neuropatía diabética se resumen en (a) daño oxidante, (b) daño osmótico, e (c) inflamación. Ver el texto. Abreviaturas: $\mathrm{ALR}=$ aldosa-reductasa; $\mathrm{SDH}=$ sorbitol-deshidrogenasa; $\mathrm{ROS}=\mathrm{radical}$ oxidante derivado del oxígeno; RNS= radical nitrosilante; GSH= glutación reducido; GSSG= glutatión oxidado; G6P= glucosa6-fosfato; R6P= ribulosa-6-fosfato; $\mathrm{F} 6 \mathrm{P}=$ fructosa-6-fosfato. $\mathrm{GAP}=$ fosfo-gliceraldehído; $\mathrm{DHAP}=$ di-hidroxi-aceton-fosfato; $\mathrm{G} 3 \mathrm{P}=$ gliceraldehído-3-fosfato; lysoPA = ácido liso-fosfatídico; PA= acil-fosfato; DAG = diacil-glicerol; PKC= proteína-kinasa-C; UDPGIcNAc = Uridín -Difosfato-N-acetilglucosamina; TGF- $\beta 1$ = transforming-growth factor beta-uno; NF- $\kappa \beta=$ Nuclear Factor Kappa beta ${ }^{11}$.

conocidos de la circulación sanguínea en el sistema nervioso periférico, vale decir, (a) el doble sistema arteriolar y (b) el fenómeno de la "válvula venosa". a) El doble sistema arteriolar (Figura 3): La arteriola extrínseca sigue un curso paralelo al fascículo, emitiendo capilares que irrigan el tejido nervioso en sentido de proximal a distal. La arteriola intrínseca penetra al fascículo nervioso desde distal a proximal. Así se asegura que las ramas nerviosas más distales tengan una irrigación arterial tan buena como las proximales ${ }^{13}$, de modo que resultaría difícil que la microangiopatía diabética por sí sola pueda producir daño isquémico en el sistema nervioso periférico.

b) La "válvula venosa". Cuando una vénula atraviesa el perineuro (Figuras 4-A y 4-B) lo hace en un ángulo oblicuo, de modo que un pequeño aumento de la presión del endoneuro, produce una obstrucción parcial del flujo venoso, limitando la circulación y produciendo isquemia. Este mecanismo adquiere importancia en los nervios periféricos que atraviesan canales osteofibrosos inextensibles, como los canales carpiano y tarsiano, y también los trayectos de nervios craneales (ver más adelante).

\section{La glicosilación de proteínas en las neuropatías}

En 1912, Maillard describió la formación de substancias de color marrón (melanoidinas) por la reacción no-enzimática entre azúcares y aminoácidos ${ }^{14}$. Desde 1981 se sabe que esta "reacción de Maillard" (sinónimo de glicosilación), también ocurre en el cuerpo humano ${ }^{15}$, donde después de décadas se acumulan proteínas irreversiblemente glicosiladas denominadas "productos de glicosilación avanzada" (AGE, por sus siglas en inglés). Esto se intensifica en personas diabéticas descompensadas, acumulándose AGE en proteínas de larga vida, como en el cristalino del ojo, en el colágeno de las membranas basales, y en el componente proteico de la mielina en el sistema nervioso periférico.

La Figura 5-A muestra que la glicosilación de 


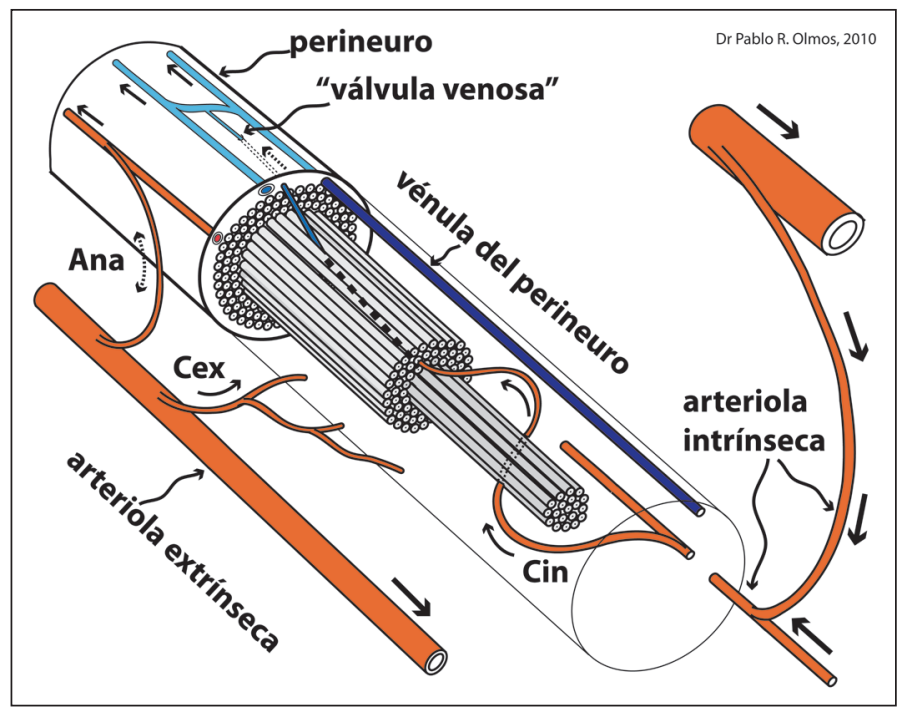

Figura 3. Anatomía microscópica, semiesquemática, de la irrigación de un nervio periférico. Se observa el doble sistema que asegura la irrigación arterial: (a) Arteriola extrínseca, que proporciona capilares (Cex) cuyo flujo va de proximal hacia distal, y (b) arteriola intrínseca, de donde emergen capilares (Cin) cuyo flujo va desde distal a proximal. Existen anastomosis (Ana) entre los dos tipos de arteriola. Se observa la "válvula venosa" de una vénula profunda antes de conectarse a una vénula del perineuro (Ilustración del autor principal, basada en ${ }^{13}$.

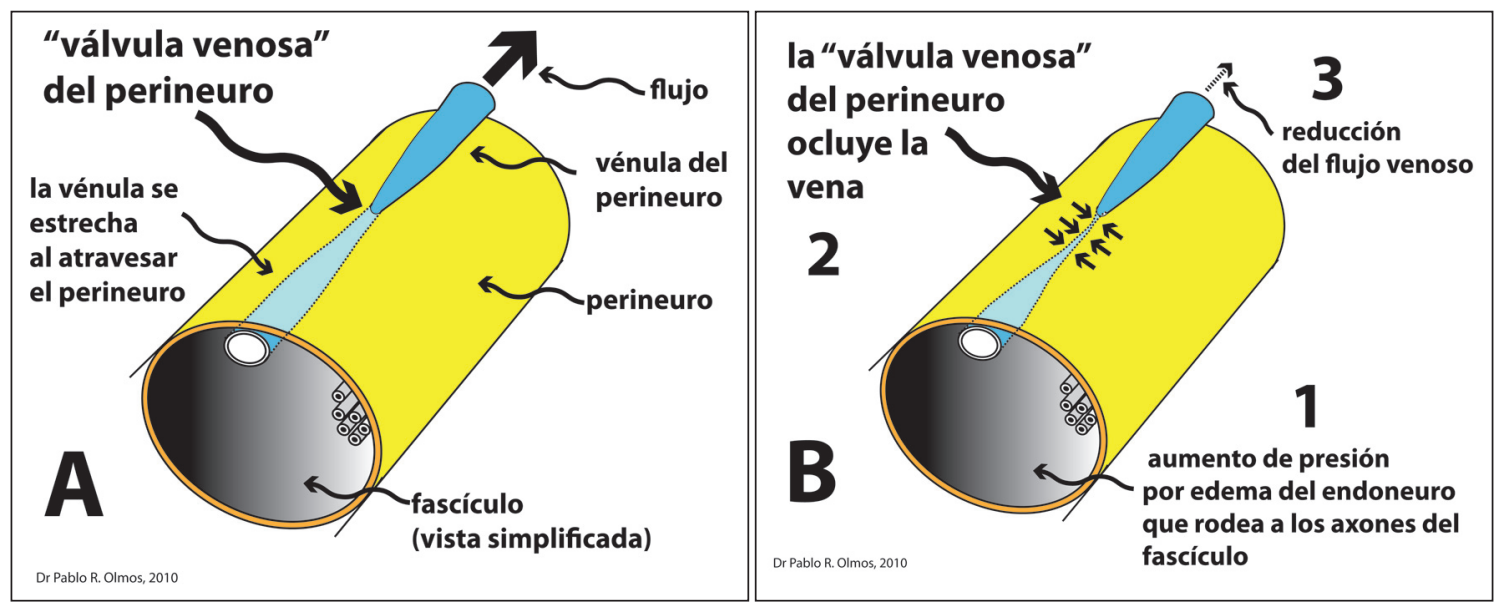

Figura 4. El mecanismo de "válvula venosa" hace a los nervios periféricos susceptibles de desarrollar edema del endoneuro. [A] Anatomía microscópica semiesquemática de una vénula del endoneuro estrechándose al atravesar el perineuro. [B] Cualquier aumento de presión (acumulación de agua) en el endoneuro comprime la vena y estira el perineuro, cerrando la "válvula venosa, lo que resulta en un empeoramiento del edema del endoneuro (Ilustración del autor principal, basada en ${ }^{13}$.

proteínas puede dividirse en tres fases-iniciación, propagación y AGE.

En la iniciación, la glucosa reacciona con los residuos epsilon-ámino $\left(--\mathrm{NH}^{+}\right)$del aminoácido lisina, formando una Base de Schiff, que se transforma en un "Producto Amadori". Un ejemplo de producto Amadori es la hemoglobina glicosilada ${ }^{16}$. El producto Amadori tiene dos destinos posibles. Si la glicemia se normaliza, entonces éste se des-glicosila, desprendiendo una molécula de proteína sin daño alguno. Sin embargo, la glucosa desprendida del producto Amadori no vuelve a ser glucosa, sino que se transforma en radicales libres violentamente oxidantes ${ }^{17}$. Si por otro lado, la hiperglicemia persistiese, entonces el producto Amadori sufriría nuevas oxidaciones, como se describe en la fase siguiente.

La fase de propagación parte con la formación de los dicarbonilos glioxal y 3-deoxiglucosona, productos de la des-glicosilación de parte del producto Amadori, y que también son potentes agentes oxidantes, capaces de catalizar nuevas 


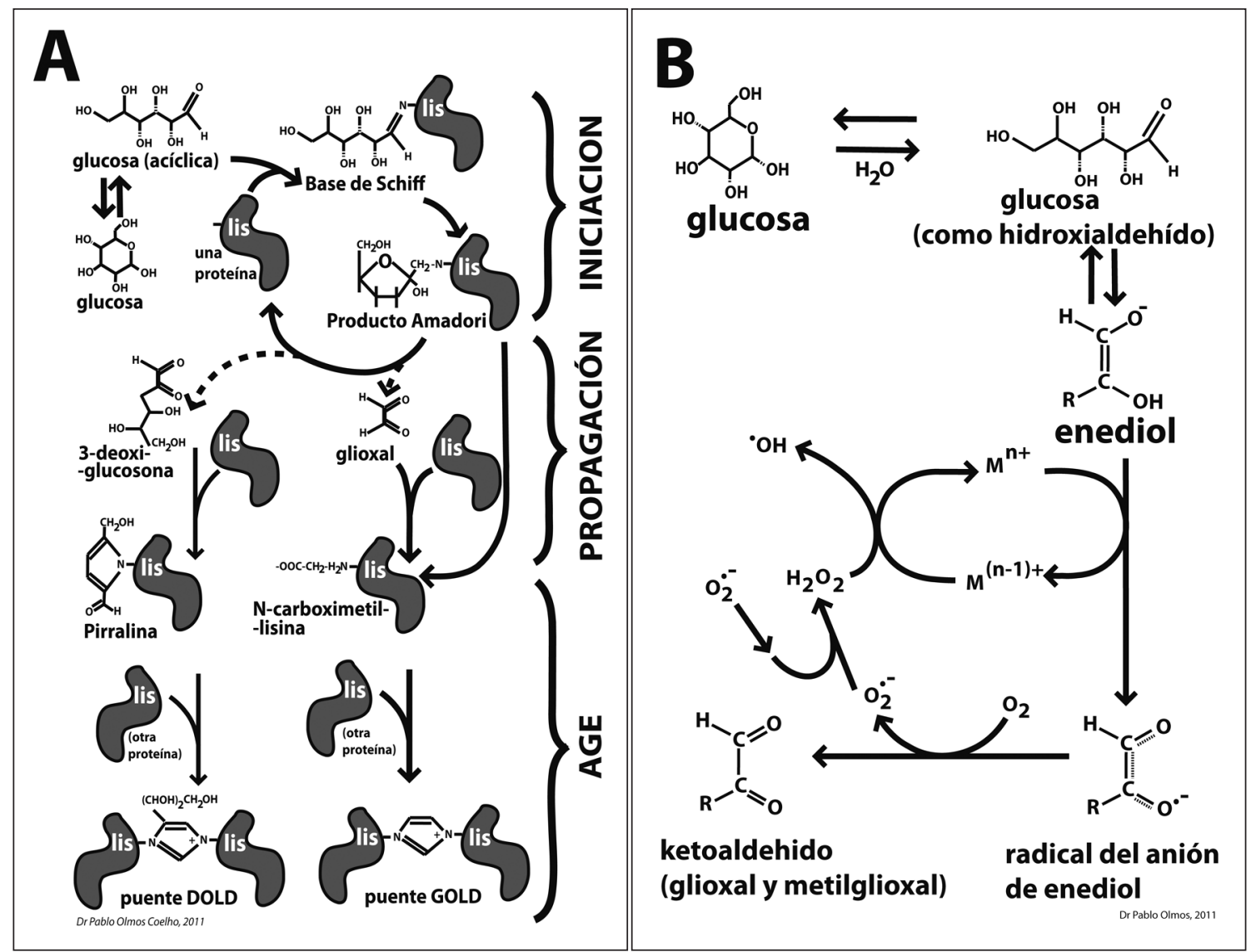

Figura 5. Glicosilación no enzimática de proteínas y Autooxidación de la Glucosa ${ }^{16,17}$. [A] Las moléculas de proteína, representadas por las figuras grises en forma de gusano, pueden glicosilarse en los residuos épsilon-ámino del aminoácido lisina. Con el tiempo, la proteína glicosilada puede llegar a tomar la forma de al menos seis tipos de "productos de glicosilación avanzada, también llamados 'AGE', por sus siglas en inglés. Dos de los AGE se ven aquí (puente DOLD y puente GOLD). [B] En presencia de metales de transición, la glucosa se autooxida, produciendo radicales libres oxidantes derivados del oxígeno ( $\mathrm{OH}=$ hidroxilo; $\mathrm{O}_{2}{ }^{-\cdots}$ = anión superóxido), $\mathrm{H}_{2} \mathrm{O}_{2}$ y ketoaldehídos. Estos últimos -glioxal y metilglioxal- también se producen como propagadores en la glicosilación de proteínas ${ }^{21}$.

reacciones tendientes a formar productos de glicosilación que, como están unidos a una sola proteína, no forman puente entre dos de ellas (pirralina y $\mathrm{N}$-carboximetil-lisina $)^{17}$.

La fase de AGE comienza con la unión de la pirralina y de la $\mathrm{N}$-carboximetil-lisina con una segunda proteína, formando "AGEs nofluorescentes pero que forman puente", llamados "puente DOLD" y "puente GOLD", con lo que se alteran irreversiblemente las estructuras terciaria y cuaternaria de las proteínas ${ }^{17-19}$.

Además de los puentes DOLD y GOLD, también se generan AGE que forman puentes entre la lisina de una proteína con la arginina de otra. Entre ellos están el "puente glucosespano” y el "puente pentosidina”. Este último es además fluorescente.

En los nervios periféricos, la glicosilación del componente proteico de la mielina hace a ésta apetecible para ser fagocitada por macrófagos que tienen receptores de AGE (RAGE) ${ }^{11,20}$, contribuyendo así, junto con la glicosilación de la tubulina (lo que altera el transporte axonal) a la génesis de la neuropatía diabética.

\section{La auto-oxidación de la glucosa y su relación con la glicosilación}

En un proceso de "auto-oxidación" (Figura 5 -B) en presencia de iones de hierro y cobre ${ }^{21}$, la glucosa en forma de hidroxialdehído se transforma en un anión radical de enediol, molécula inestable, 


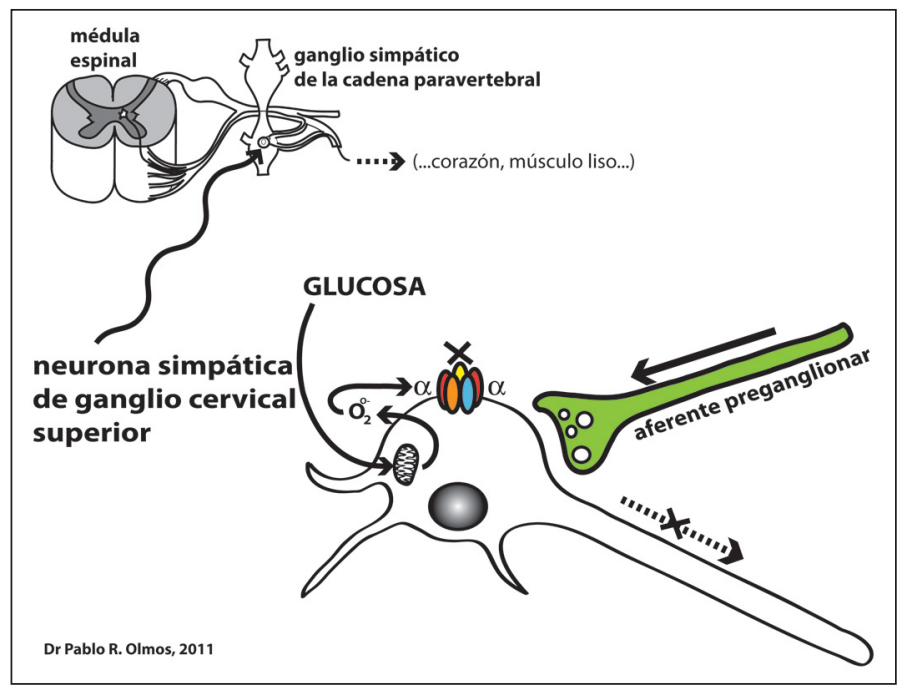

Figura 6. Un caso particular de daño neuronal oxidante - Neuropatía autonómica: En una neurona post-ganglionar de la cadena simpática, los radicales libres oxidan el componente alfa del canal de sodio, inutilizándolo e impidiendo así que la célula se depolarice aún cuando reciba potenciales de acción provenientes del aferente preganglionar22. que al transformarse a su vez en un ketoaldehído, genera ión superóxido, agua oxigenada y radical hidroxilo. Por su parte, el ketoaldehído es el mismo glioxal de la Figura 5-A, arriba (o bien metilglioxal cuando el $\mathrm{R}=-\mathrm{CH}_{3}$ ).

Estos mecanismos de estrés oxidante se suman al mecanismo ya mencionado en los aspectos metabólicos, arriba y en la Figura 2, donde el consumo de NADPH reduce la actividad del ciclo de regeneración del glutatión.

\section{La oxidación en el caso particular de la neuropatía autonómica}

La neurona autonómica tiene un mecanismo propio de daño en la diabetes mellitus.

En efecto, una neurona autonómica (simpática) postganglionar típica tiene canales de sodio en la membrana plasmática. Como podemos ver en la Figura 6, en el caso de la diabetes mellitus crónicamente descompensada, el componente alfa del canal de sodio se oxida, de modo que la neurona postganglionar no es capaz de generar sus propios potenciales de acción. Esta oxidación se debe a que en condiciones de hiperglicemia, en la neurona autonómica se intensificarían los fenómenos químicos debidos al déficit de NADPH ilustrados en la Figura 2, arriba ${ }^{22}$.

\section{Factores de crecimiento neuronal y neuropatía precoz}

En modelos animales de DM1, a medida que disminuye gradualmente la producción de insu- lina, pero antes de que se desencadene la hiperglicemia, ya hay signos de neuropatía periférica ${ }^{5}$. A primera vista, esto pudiese parecer una inconsistencia, a la luz de lo ya comentado sobre el papel de la hiperglicemia en la génesis de la ND. Para desentrañar este aparente contrasentido, remitámonos a la Figura 7, que explica como la insulina influye en la salud axonal normal ${ }^{5,23}$, a través de "factores de crecimiento neuronal" (NGF, por sus siglas en inglés), que interactúan con dos tipos de receptores en la neurona, Trk-A y P75-NTR, para así regular el crecimiento, desarrollo, supervivencia y reparación del sistema nervioso periférico. La unión del NGF con el receptor Trk-A produce la trans-fosforilación de éste, lo que resulta en el reclutamiento de una serie de proteínas a sitios de unión del receptor, con lo que se activan las MPP-Kinasas a través del sistema Raf. Las vías que incluyen MEK1y2 y ERK1y2 estimulan el crecimiento neuronal. Por su parte, las vías que usan RSK vía ERK, y Akt vía PI3-Kinasa estimulan la supervivencia neuronal, a través de la subsecuente activación de Bcl-2 e inhibición de p53. Todos estos mediadores son estimulados a través de la acción de la insulina sobre los factores de crecimiento neuronal.

En contraposición a la recién descrita vía del receptor Trk-A, la vía del receptor P75-NTR podría llevar a la apoptosis neuronal por sus propios mecanismos, aunque afortunadamente la vía del receptor Trk-A logra silenciarlos.

Por otro lado, la apoptosis neuronal es estimu- 


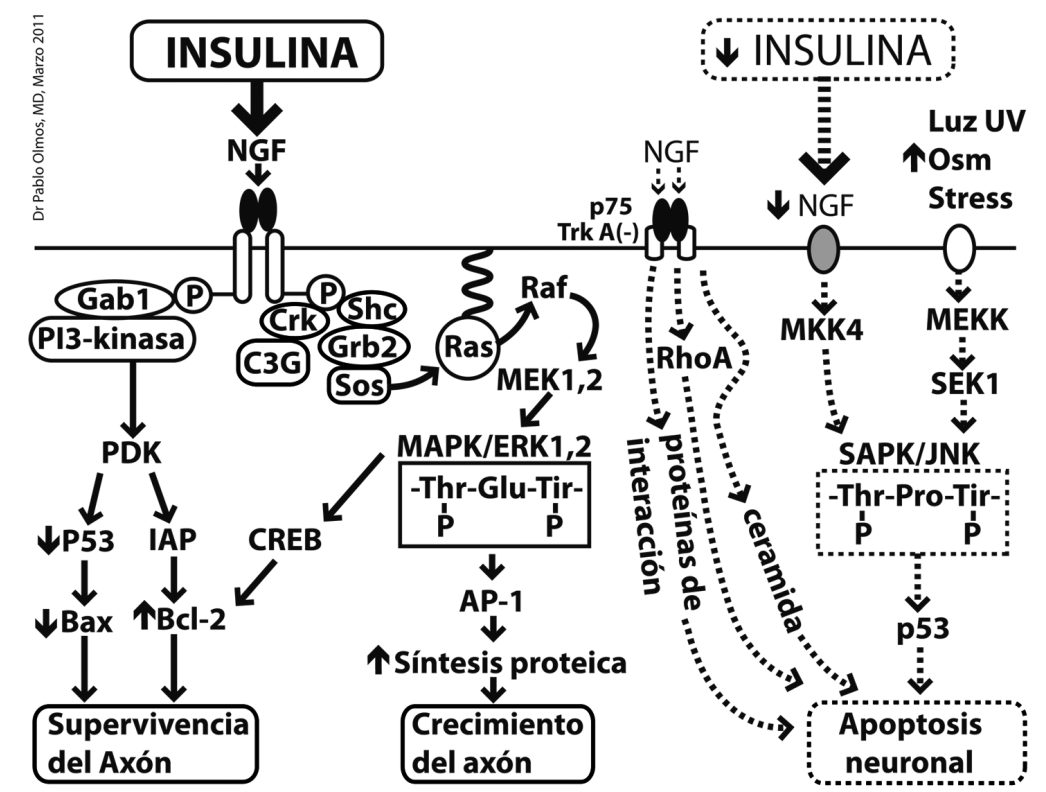

Figura 7. Influencia de la insulina sobre la salud axonal aún antes de que exista hiperglicemia (dibujo basado en [5,23-27]). [a] A la izquierda de la figura, puede verse como una concentración normal de insulina es indispensable para la supervivencia y el crecimiento del axón. [b] A la derecha se resume cómo el déficit relativo de insulina contribuye a la apoptosis neuronal (vea el texto). Abreviaturas: AP-1 = proteína activadora-1, es un factor de transcripción capaz de regular la expresión genética en respuesta a estímulos; $\mathbf{B a x}=$ BCL2-associated-X-protein, es un acelerador de apoptosis; $\mathbf{B C L}-\mathbf{2}=$ molécula supresora de apoptosis; C3G = factor de intercambio guanina-nucleótido; $\mathbf{C R E B}=$ cAMP-response element-binding, se une a ciertas secuencias del DNA, modulando la expresión de genes aguas-abajo de éstas; $\mathbf{C r k}=$ molécula adaptadora que se une a proteínas tirosina-fosforiladas; IAP = inhibidor de apoptosis; MAPK (mitogen-activated proteína kinasa) y ERK (extracellular-signal-regulated kinase) intervienen

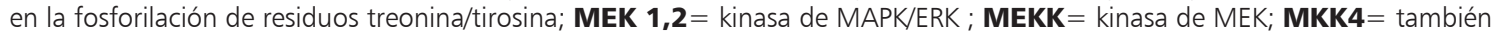
llamado MEK4, cuando se activa por MAPKKKs, se fosforila y activa a su vez JNKs que regulan desarrollo neuronal, apoptosis, inflamación y respuestas celulares al stress; NGF= Nerve Growth Factor; Shc = lo mismo que el Gab-1 y el Grb2, son proteínas intermediarias entre la señal del receptor Trk-A y la vía de transducción RAS/PI3 kinasa/Akt ; $\mathbf{P}=$ fosfato; $\mathbf{p 5 3}=$ factor de transcripción nuclear de 43,7 KDa; $\mathbf{P 7 5 N T R}=$ receptor de alta afinidad del NGF ; PI3-kinasa = fosfatidil-inositol-3-kinasa; p75 = receptor de neurotrofina (Roux PP, 2001); PDK= Piruvato Deshidrogenasa Kinasa; Sos= molécula activadora del Ras; Ras= es una "proteína-G", llamada así porque se activa uniéndose al GTP, el que es hidrolizado a GDP, el cual debe ser liberado y reemplazado por nuevo GTP para que el Ras se active de nuevo; RhoA=GTP-asa de la familia Ras-homóloga; $\mathbf{R a s =}$ kinasa de MAPK ; SAPK/JNK = Stress-activated protein kinase/c-Jun $\mathrm{NH}_{2}$-terminal kinase ; SEK-1= stress-activated protein kinase; Trk-A = receptor de alta afinidad del NGF.

lada también vía MKK4-Bax por la disminución de los NGF a consecuencia de la disminución de la acción insulínica.

La Tabla 1 muestra un resumen en el que se observa la tendencia a la disminución de cinco factores de crecimiento neuronal y sus receptores en modelos animales de diabetes mellitus.

\section{Factores inflamatorio-inmunológicos y neuropatía}

Las neuropatías diabéticas de fisiopatología predominantemente inflamatorio-autoinmune no sólo se limitan a la radículo-plexopatía lum- bosacra, sino que también incluyen a la radículoplexopatía cervical, a la radiculopatía torácica, y a las neuropatías craneales. En efecto, la Figura 8 muestra la pérdida axonal y la infiltración leucocitaria que ocurre en este tipo de neuropatías.

En las neuropatías diabéticas de fisiopatología predominantemente inflamatorio-autoinmune, el metabolismo intraneuronal de la glucosa (ver nuevamente Figura 2) se desviaría principalmente hacia la producción de citokinas pro-inflamatorias y fibrogénicas, como el NF-k $\beta$ (Nuclear Factor Kappa beta) y el TGF $\beta$ (Transforming Growth Factor-beta) ${ }^{4,12}$. 

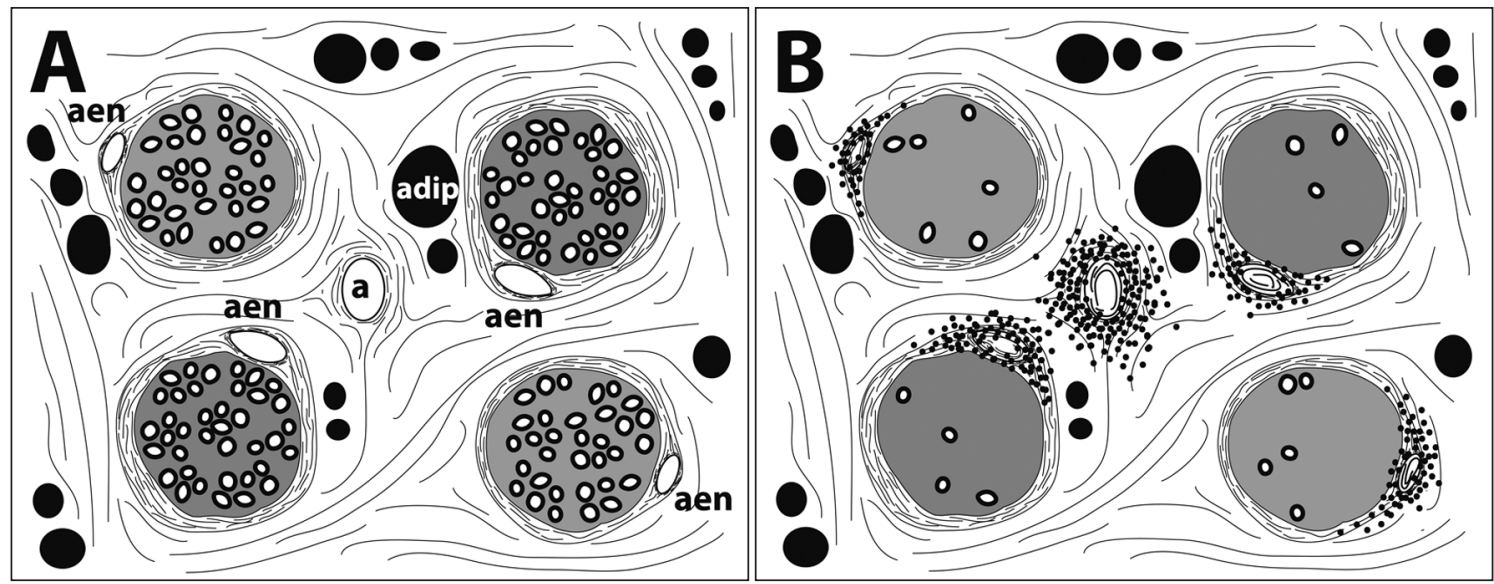

Figura 8. Mecanismos inflamatorio-inmunológicos en algunas neuropatías diabéticas. [A] Figura semiesquemática de la histología normal de un nervio periférico con fibras mielinizadas. Se observan cuatro fascículos conteniendo axones mielinizados. En el borde de cada fascículo hay una arteriola del endoneuro (aen). En el tejido conectivo entre los fascículos hay una arteriola (a) y varias células adiposas (adip). [B] Neuropatía Diabética de Fisiopatología predominantemente Inflamatorio-inmunológica (radículo-plexopatías lumbosacra y cervical, radiculopatía torácica, y neuropatía craneal). Se observa una casi total pérdida axonal y la infiltración inflamatoria de las arteriolas del endoneuro con abundantes leucocitos [dibujo basado en 4, Tracy JA, 2008)].

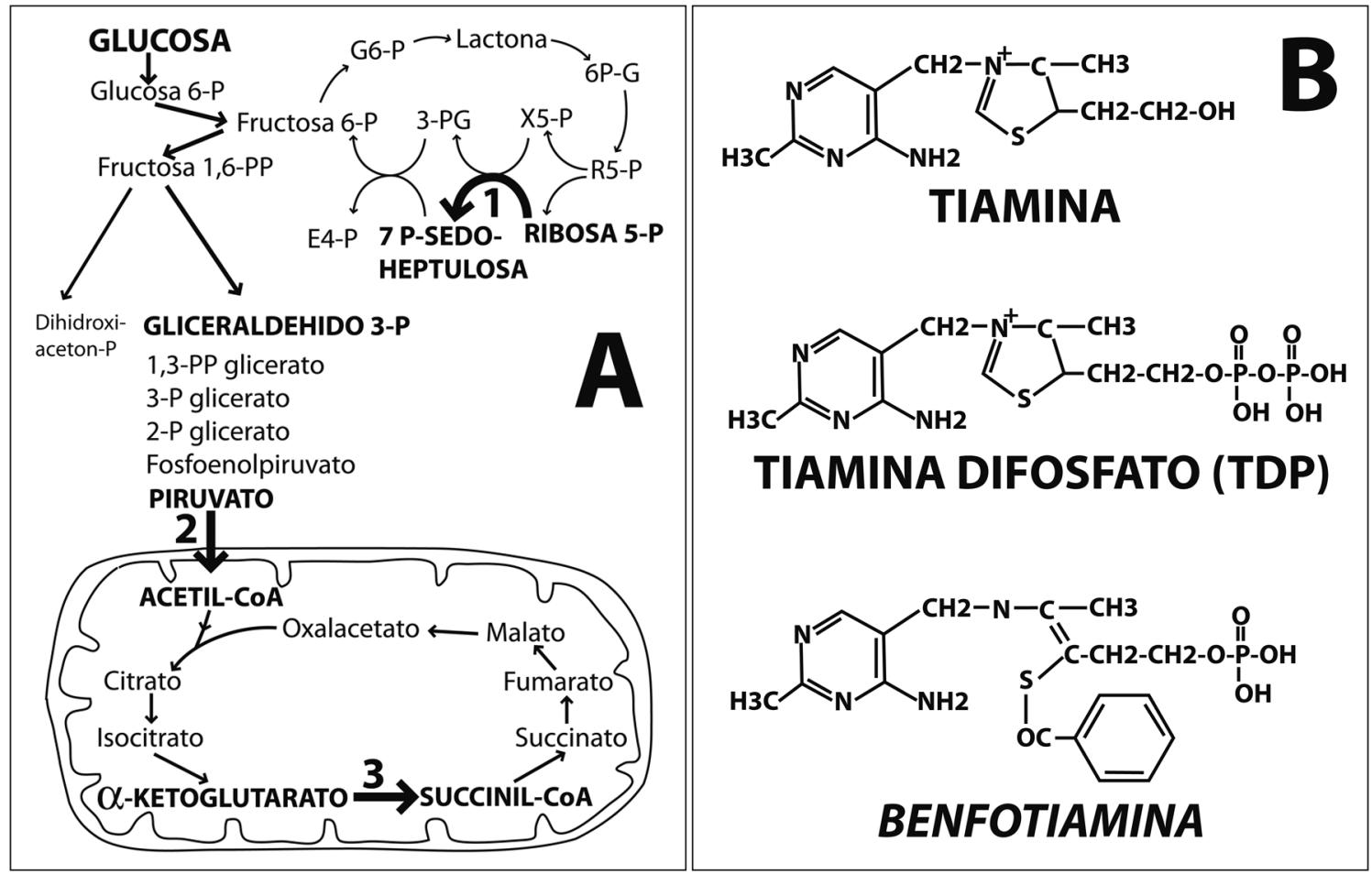

Figura 9. [A] esquema del metabolismo de la glucosa. Los tres números destacan las tres reacciones bioquímicas donde interviene la tiamina como cofactor: [1] Transketolasa en el ciclo de las pentosas; [2] Piruvato Deshidrogenasa en la glicolisis del citosol;[3] a-Ketoglutarato deshidrogenasa en el ciclo de Krebs de la mitocondria. [B] las tres estructuras químicas de la tiamina. Observe que la palabra "Benfotiamina" está escrita en cursiva, porque no es un producto natural sino una modificación farmacológica de la tiamina (ver el texto) ${ }^{28}$. Abreviaturas: $\mathbf{G 6 - P}=$ glucosa-6-fosfato; $\mathbf{6 P - G}=$ 6-fosfogluconato; $\mathbf{R 5}-\mathbf{P}=$ ribulosa5-fosfato; X5-P= xilulosa-5-fosfato; 3P-G = 3-fosfoglicerato; E4-P= eritrosa-4-fosfato. 
Fisiopatología neuropatía diabética - P. R. Olmos et al

Tabla1. Concentración de neurotrofinas y expresión de sus receptores en modelos animales de diabetes ${ }^{5}$

\begin{tabular}{|c|c|c|c|}
\hline Neutrofinas & Estado en diabetes & $\begin{array}{l}\text { Receptores de } \\
\text { neurotrofinas }\end{array}$ & Estado en diabetes \\
\hline $\begin{array}{l}\text { nerve Growth Factor (NGF) } \\
\text { Suero } \\
\text { Piel } \\
\text { Nervio } \\
\text { Corazón }\end{array}$ & $\begin{array}{l}\downarrow \\
\downarrow \\
\downarrow \\
\downarrow\end{array}$ & $\begin{array}{l}\text { Trk-A } \\
\text { DRG }\end{array}$ & $\downarrow$ \\
\hline $\begin{array}{l}\text { Brain-Derived Neurotrophic Factor (BDNF) } \\
\text { Músculo } \\
\text { Nervio }\end{array}$ & $\begin{array}{l}\downarrow \\
\downarrow\end{array}$ & Trk-B & -------- \\
\hline $\begin{array}{l}\text { Neurotrphin-3 } \\
\text { Músculo } \\
\text { Piel } \\
\text { Nervio }\end{array}$ & $\begin{array}{l}\downarrow \\
\vec{\downarrow} \\
\downarrow\end{array}$ & $\begin{array}{l}\text { Trk-C } \\
\text { DRG }\end{array}$ & $\downarrow$ \\
\hline $\begin{array}{l}\text { Neurotrophin 4/5 } \\
\text { Nervio }\end{array}$ & $\downarrow$ & Trk-B & ---------- \\
\hline
\end{tabular}

Tabla 2. Clasificación fisiopatológica actual de las neuropatías diabéticas ${ }^{19}$

\begin{tabular}{|c|c|c|}
\hline Fisiopatología & Subtipo clínico de neuropatía & Mecanismo(s) \\
\hline $\begin{array}{l}\text { Metabólico- } \\
\text { microvascular-hipóxica }\end{array}$ & $\begin{array}{l}\text { - Polineuropatía distal (DPN)* } \\
\text { - Neuropatía autonómica (DAN) }\end{array}$ & $\begin{array}{l}\text { Figuras } 1,2 \text { y } 5 \text { (y } 7 \text { cuando es precoz) } \\
\text { Figuras } 1,2,5 \text { y } 6\end{array}$ \\
\hline Inflamatorio-autoinmune & $\begin{array}{l}\text { - Radículo-plexopatía lumbosacra (DLRPN) } \\
\text { - Radículo-plexopatía cervical (DCRPN) } \\
\text { - Radiculopatía torácica (DTRN) })^{\dagger+} \\
\text { - Neuropatía craneal } \\
\text { - Neuropatía dolorosa + baja de peso }\end{array}$ & $\begin{array}{l}\text { Figuras } 2 \text { y } 8 \\
\text { Figuras } 2 \text { y } 8 \\
\text { Figuras } 2 \text { y } 8 \\
\text { Figuras } 2 \text { y } 8 \\
\text { Figuras } 2 \text { y } 8\end{array}$ \\
\hline Compresiva & $\begin{array}{l}\text { - Síndrome del canal carpiano } \\
\text { - Neuropatía ulnar del codo (UNE) })^{\S \S} \\
\text { - Neuropatía peronea }\end{array}$ & $\begin{array}{l}\text { Figuras } 3 \text { y } 4 \\
\text { Figuras } 3 \text { y } 4 \\
\text { Figuras } 3 \text { y } 4\end{array}$ \\
\hline $\begin{array}{l}\text { Secundarias a complica- } \\
\text { ciones de la diabetes }\end{array}$ & $\begin{array}{l}\text { - N. por Ketoacidosis } \\
- \text { N. por insuficiencia renal } \\
\text { - N. por isquemia de grandes vasos }\end{array}$ & $\begin{array}{l}\text { ¿Coagulación Intravascular? } \\
\text { Síndrome urémico } \\
\text { Isquemia extrema }\end{array}$ \\
\hline $\begin{array}{l}\text { Relacionada con trata- } \\
\text { miento }\end{array}$ & $\begin{array}{l}\text { - Neuritis Insulínica [muy rara] } \\
\text { - Neuropatía hipoglicémica [muy rara] }\end{array}$ & $\begin{array}{l}\text { Isquemia por apertura de puentes arteriovenosos } \\
\text { [descrita en insulinomas] }\end{array}$ \\
\hline
\end{tabular}

*Por sus siglas en inglés: "Diabetic PolyNeuropathy". 'Por sus siglas en inglés: "Diabetic Autonomic Neuropathy". \$Por sus siglas en inglés: "Diabetic Lumbosacral Radiculoplexus Neuropathy". "Por sus siglas en inglés: "Diabetic Cervical Radiculoplexus Neuropathy". "tPor sus siglas en inglés: "Diabetic Thoracic Radiculopathy". ${ }^{\S}$ Por sus siglas en inglés: "Ulnar Neuropathy at the Elbow".

\section{Clasificación clínico-fisiopatológica actual}

La Tabla 2 muestra la clasificación fisiopatológica actual de la neuropatía, a la que hemos agregado referencias a las figuras que ilustran sus mecanismos íntimos.

Las neuropatías metabólico-microvasculares son las más frecuentes. La polineuropatía distal (DPN, por sus siglas en inglés) se caracteriza por disminución de la sensibilidad térmica-dolorosa, más severa en las regiones más distales de las extremidades. Su severidad aumenta con el empeoramiento del control glicémico. Algo similar sucede con la neuropatía autonómica, donde a los mecanismos metabólico-microvasculares de la DPN se agrega la glicosilación de los canales de sodio ya mencionados.

Entre las neuropatías inflamatorio-autoinmu- 
nes, la radículo-plexopatía lumbosacra (DLRPN por sus siglas en inglés) ocurre en $1 \%$ de los pacientes con dolor de extremidades inferiores, pérdida de fuerza y atrofia del cuádriceps. Sus sinónimos son "amiotrofia diabética", "síndrome de Bruns-Garland" "monoreuritis multiplex diabética" "poliradiculopatía diabética" y "neuropatía diabética proximal"10. El líquido cefalorraquídeo en la DLRPN tiene elevación de proteínas (promedio $89 \mathrm{mg} / \mathrm{dL}$ ). Tiene tendencia a la mejoría con tratamiento sintomático del dolor, rehabilitación y control glicémico óptimo con insulino-terapia intensificada, sin que el agregado de glucocorticoides sea de utilidad ${ }^{4,12}$. Algo similar sucede con la radículo-plexopatía cervical, la radiculopatía torácica, y las neuropatías craneales.

Las neuropatías compresivas tienen en común un aumento de volumen de axones y fascículos, lo que causa daño isquémico cuando nervio atraviesa canales osteofibrosos inextensibles, como el canal del carpo (síndrome de canal carpiano), la epitróclea (neuropatía ulnar) y la cabeza del peroné (neuropatía peronea). En estos casos, el mecanismo de "válvula venosa" (Figuras 4-A y 4-B) ya mencionado es el que predominaría, sobre la base del edema axonal causado por acumulación de sorbitol (Figura 2).

De las neuropatías relacionadas con complicaciones de la diabetes, la que ocurre luego de recuperarse de una ketoacidosis es excepcionalmente rara, sólo mencionada en 1974 en 6 autopsias de pacientes que murieron luego de una ketoacidosis, y que presentaban lesiones isquémicas en cerebro y otros órganos, atribuibles a coagulación intravascular $^{12,28}$.

Con respecto a las neuropatías relacionadas con el tratamiento de la diabetes, destaca la "neuritis insulínica", descrita en 1933, que consiste en una neuropatía extremadamente dolorosa, precedida por una brusca mejoría del control glicémico mediante insulina ${ }^{29,30}$ al reanudar tratamiento insulínico que había sido suspendido con el fin de perder peso, lo que sugiere déficit de tiamina (ver más adelante) más que la isquemia sugerida en modelos animales ${ }^{30-32}$.

Finalmente, la neuropatía asociada a hipoglicemia ha sido descrita no en pacientes diabéticos, sino en pacientes con insulinoma-tumores pancreáticos productores de insulina. En ratas tratadas con inyecciones de insulina, sólo aquellas que sufrieron hipoglicemia prolongada (y no las que sufrieron sólo hiperinsulinemia sin hipoglicemia) mostraron daño en fibras motoras y en neuronas del asta anterior medular ${ }^{4,33}$.

\section{El déficit de tiamina (vitamina B1) y las neuropatías diabéticas}

Aunque este artículo es sobre fisiopatología y clasificación de la ND, y no sobre tratamiento, mencionamos a la tiamina, cuyo déficit -que es más frecuente de lo que comúnmente se piensaestá involucrado en la génesis del daño del sistema nervioso periférico en la diabetes mellitus.

La tiamina (vitamina $\mathrm{B}_{1}$ ) se absorbe en el tubo digestivo, y como tiamina-difosfato (TPP), actúa como coenzima en tres etapas del metabolismo de la glucosa: Transketolasa en el ciclo de las pentosas, piruvato deshidrogenasa en la glicolisis del citosol, y a-Ketoglutarato deshidrogenasa en el ciclo de Krebs de la mitocondria. Un déficit de tiamina reduciría drásticamente: (a) la génesis de ribosa y desoxiribosa (necesarias para la síntesis de ácidos nucleicos), (b) la producción de NADPH (necesario para la regeneración del glutatión y síntesis de ácidos grasos), y (c) la producción aeróbica de $\mathrm{ATP}^{34-36}$.

La tiamina se excreta por filtración glomerular, y se reabsorbe en el túbulo proximal. La deficiencia severa produce los conocidos cuadros de Beriberi (que incluye una severa neuropatía periférica sensitivo-motora) $)^{37}$, y los síndromes de Wernike (ataxia) y de Korsakoff (encefalopatía amnésica).

Existe una alta frecuencia (17-79\%) de déficit de tiamina en diabéticos, debido a una menor absorción intestinal ocasionada por el déficit de acción insulínica. A su vez, el mismo déficit de tiamina reduce la producción de insulina en la célula beta pancreática. Por último, en diabéticos la reabsorción tubular proximal de tiamina no aumenta compensatoriamente en situación de déficit.

En diabéticos con déficit de tiamina, la frenación del ciclo de las pentosas aumenta la proporción de glucosa que sigue la vía de la fructosa 1,6 di-fosfato (Figura 9), que a su vez incrementa la producción de proteína kinasa-C, intermediario en la patogenia de retinopatía, nefropatía ${ }^{38}$ y neuropatía (Figura 2) diabéticas. Por otro lado, la reducción del NADPH disminuye aún más la defensa antioxidante-antiglicosilante (Figuras 2 y 5$)^{39}$. 
Se recomienda agregar tiamina (o su derivado lipofílico benfotiamina) al tratamiento de neuropatía diabética. En forma preventiva, se recomienda incluir en el tratamiento de los diabéticos más de $4 \mathrm{mg}$ diarios por vía oral de vitamina $\mathrm{B}_{1}$, que es una dosis mayor que la recomendación diaria para la población general (1-1,4 mg/día $)^{39-41}$.

\section{La importancia del control glicémico}

Luego de la lectura de este artículo, el lector quizá esté esperando una sección referente al tratamiento farmacológico de la ND.

En realidad no es este el objetivo de este trabajo.

De hecho, junto con una mejor comprensión de la nueva clasificación fisiopatológica de ND, hay un mensaje de fondo que no podemos dejar de transmitir-que el control glicémico óptimo (actualmente considerado como hemoglobina glicosilada bajo $7,0 \%)^{42}$ es la vía fundamental para [a] prevenir y [b] tratar cualquier forma de ND. Este mensaje proviene del conocimiento que todas las vías metabólico -moleculares que hemos analizado, tienen un solo punto de partida- la hiperglicemia. En efecto, en DM1, los estudios DCCT-EDIC ${ }^{42}$ demostraron que el control intensivo de la glicemia por una media de 6,5 años, reduce la aparición de neuropatía diabética en $64 \%$. Por otro lado, en DM2, el estudio UKPDS mostró una reducción de 39,6\% en neuropatía diabética en pacientes con tratamiento intensivo de la glicemia ${ }^{43}$.

Los comentarios del párrafo precedente se aplican a la prevención primaria y secundaria de la ND.

Con respecto del tratamiento de la ND ya establecida, fuera de la mejoría de la glicemia y la reposición de Tiamina, los demás tratamientos farmacológicos de la ND están orientados fundamentalmente a bloquear los síntomas (dolor, parestesias), y son en este sentido muy útiles, pero no son capaces por sí solos de evitar ni reparar ni evitar que continúe el daño del sistema nervioso periférico causado por la hiperglicemia. Los tratamientos farmacológicos de los síntomas de ND serán materia de otro artículo.

\section{Conclusión}

Las neuropatías diabéticas, un conjunto de diversas manifestaciones de daño del sistema nervioso periférico y autonómico en pacientes con diabetes mellitus, ocurren a consecuencia de la hiperglicemia crónica, la que actúa a través de una serie de mecanismos fisiopatológicos que llevan a un daño no sólo funcional sino también estructural y molecular de las fibras nerviosas. Junto con los siempre necesarios tratamientos sintomáticos, es indispensable agregar a la terapia de cualquier neuropatía diabética el control estricto de la glicemia. Finalmente, es necesario tener presente una adecuada suplementación de tiamina no sólo para el tratamiento sino que también para la prevención de la neuropatía.

\section{Referencias}

1. Said G, Baudoin D, Toyooka K. Sensory loss, pains, motor deficit and axonal regeneration in length-dependent diabetic polyneuropathy. J Neurol 2008; 255: 1693-702.

2. Sima AAF. New insights into the metabolic and molecular basis for diabetic neuropathy. Cell Mol Life Sci 2003; 60: $1-20$.

3. Skljarevski V. Historical aspects of diabetic neuropathies. En: Veves A, Malik RA, Editors. Diabetic neuropathy, clinical management. 2007 by Humana Press Inc., New Jersey, USA. Páginas 1-5.

4. Tracy JA, Dick PJB. The spectrum of diabetic neuropathies. Phys Med Rehabil Clin N Am 2008; 19 (1): 1-26. Doi:10.1016/j.pmr.2007.10.010.

5. Yasuda H, Terada M, Maeda K, Kogawa S, Sanada M, Haneda M, et al. Diabetic neuropathy and nerve regeneration. Progress in Neurobiology 2003; 69: 229-85.

6. Iribarren B, Passi G, Aybar N, Ríos P, González L, Rojas MA, Saavedra F. Pie diabético: Evolución de una serie de 121 pacientes. Rev Chilena de Cirugía 2007; 59 (5): 337-41.

7. Adler AI, Erqou S, Lima TAS, Robinson AHN. Association between glycated haemoglobin and the risk of lower extremity amputation in patients with diabetes mellitus-review and meta-analysis. Diabetologia 2010; 53: 840-9.

8. Shakher J, Stevens MJ. Update on the management of diabetic polyneuropathies. Diabetes, Metabolic Syndrome and Obesity: Targets and Therapy 2011; 4: 289-305.

9. Eastman RC. Chapter 15: Neuropathy in Diabetes. En: Diabetes in America, 2nd Edition. National Diabetes Data Group. National Institutes of Health. National Institute of Diabetes and Digestive and Kidney Diseases. NIH Publication No 95-1468. Año 1995. Páginas 339-48. 
10. Sinnreich M, Taylor BV, Dick PJ. Diabetic neuropathies: Classification, clinical features and pathophysiological basis. The Neurologist 2005; 11: 63-79.

11. Bastías MJ; Toro L; Olmos P. La insulinoterapia intensificada más antineuríticos es superior a antineurítico puro en neuropatía diabética dolorosa. Rev Med Chile 2006; 134 (12): 1507-15.

12. Tracy JA, Engelstad JK, Dyck PJB. Microvasculitis in diabetic lumbosacral radiculoplexus neuroipathy. J Clin Neuromuscul Dis 2009; 11 (1): 44-8.

13. Myers RR. Anatomy and microanatomy of peripheral nerve. Neurosurg Clin N Am. 1991; 2 (1): 1-20.

14. Sakurai S, Yonekura H, Yamamoto Y, Watanabe T, Tanaka N, Li H, et al. The AGE-RAGE system and diabetic nephropathy. J Am Soc Nephrol 2003; 14: S259-63.

15. Monnier VM, Cerami A. Nonenzymatic browning in vivo: Possible process for aging in long-lived proteins. Science 1981; 211: 491-4.

16. Jakus V, Rietbrock N. Advanced glycation end-products and the progress of diabetic vascular complications. Physiol Res 2004; 53: 131-42.

17. Thornalley PJ, Langborg A, Minhas HS. Formation of glyoxal, methylglyoxal and 3-deoxyglucosone in the glycation of proteins by glucose. Biochem J 1999; 344: 109-16.

18. Ahmed N. Advanced glycation endproducts-role in pathology of diabetic complications. Diab Res Clin Pract 2005; 67: 3-21.

19. Ahmed N, Thornalley PJ. Advanced glycation endproducts : What is their relevance to diabetic complications?. Diab Obes Metab 2007; 9: 233-45.

20. Stern DM, Yan SD, Yan SF, Schmidt A-M. Receptor for advanced glycation endproducts (RAGE) and the complications of diabetes. Age Res Rev 2002; 1-15.

21. Wolff SP. Transition metals and oxidative stress in the complications of diabetes. En: Gries FA, Wessel K., editors. The role of antioxidants in diabetes mellitus. (C)1993 por Universimed Verlag, Frankfurt am Main, Alemania, páginas 82-101.

22. Diano S, Horvath TL. A sympathetic view on free radicals in diabetes. Neuron 2010; 37: 809-811; DOI 10.1016/j.neuron.2010.06.011.

23. Atwal JK, Massie B, Miller FD, Kaplan DR. The TrkB-Shc Site Signals Neuronal Survival and Local Axon Growth via MEK and PI3-Kinase. Neuron 2000; 27: 265-77.

24. Kolch W. Meaningful relationships : the regulation of the Ras/Raf/MEK/ERK pathway by protein interactions. Biochem. J. (2000) 351: 289-305.

25. Roux PP, Bhakar AL, Kennedy TE, Barkeri PA. The p75 Neurotrophin Receptor Activates Akt (Protein Kinase B) through a Phosphatidylinositol 3-Kinase-dependent
Pathway. The Journal of Biological Chemistry 2001; 276 (25): 23097-104.

26. Kolch W. Meaningful relationships: The regulation of the Ras/Raf/MEK/ERK pathway by protein interactions. Biochem J 2000; 15 (351 pt 2): 289-305.

27. Nishina H, Wada T, Katada T. Physiological Roles of SAPK/JNK Signaling Pathway. J Biochem 2004; 136 (2): 123-6.

28. Timperley WR, Preston FE, Ward JD. Cerebral intravascular coagulation in diabetic ketoacidosis. Lancet 1974; 1 (7864): 952-956 [PubMed: 4133643].

29. Caravati CM. Insulin neuritis: a case report. Va Med Monthly 1933; 59: 745-6.

30. Gibbons $\mathrm{CH}$, Freeman R. Treatment induced diabetic neuropathy-a reversible painful autonomic neuropathy. Ann Neurol 2010; 64 (4): 534-41. Doi:10.1002/ ana.21952.

31. Kihara M, Zollman PJ, Smithson IL, Lagerlund TD, Low PA. Hypoxic effect of exogenous insulin on normal and diabetic peripheral nerve. Am J Physiol 1994; 266 (6 pt 1): E980-5.

32. Tesfaye S, Mailk R, Harris N, Jakubowski JJ, Mody C, Rennie IG, et al. Arterio-venous shunting and proliferating new vessels in acute painful neuropathy of rapid glycaemic control (insulin neuriris). Diabetologia 1996; 39 (3): 329-35.

33. Sima AA, Zhang WX, Greene DA. Diabetic and hypoglycemic neuropathy-a comparison in the BB rat. Diabetes Res Clin Pract 1989; 6 (4): 279-96.

34. Niemeyer H. Capítulo 12 - Metabolismo de los hidratos de carbono. En: Niemeyer H. Bioquímica. $2^{\circ}$ Edición 1978 por Editorial Inter-Médica S.A.I.C.I., Buenos Aires, República Argentina. Páginas 33-63.

35. Nelson DL, Cox MM. Glycolysis, gluconeogenesis and the pentose phosphate pathway. In: Nelson DL, Cox MM, Editors. Leninger: Principles of Biochemistry. 5th Edition 2008 by W. H. Freeman and Company, New York, U.S.A. Pages 550-1.

36. Beltramo E, Berrone E, Tarallo S, Porta M. Effects of thiamine and benfotiamine on intracelular glucose metabolism and relevance in the prevention of diabetic complications. Acta Diabetol 2008; 45: 131-41.

37. Igata A. Clinical studies on rising and re-rising neurological diseases in Japan. Proc Jpn Acad 2010; Ser B 86: 366-76.

38. Olmos P, Araya-Del-Pino A, González C, Laso P, Irribarra V, Rubio L. Fisiopatología de la retinopatía y nefropatía diabéticas. Rev Med Chile 2009; 137: 1375-84.

39. Balakumar P, Rohilla A, Krishan P, Solairaj P, Thangathirupathi A. The multifaceted therapeutic potential of benfotiamine. Pharm Res 2010; 61: 482-8. 
Fisiopatología neuropatía diabética - P. R. Olmos et al

40. Thornalley PJ. The potential role of thiamine (vitamin B1) in diabetic complications. Curr Diabetes Rev 2005; 1 (3): 287-98.

41. Page GLJ, Laight $\mathrm{D}$, Cummings MH. Thiamine deficiency in diabetes mellitus, and the impact of thiamine replacement on glucose metabolism and vascular disease. Int J Clin Pract 2011; 65 (6): 684-90.

42. Pop-Busui R, Herman WH, Feldman EL, Low PA, Mar- tin CL, Cleary PA, et al, fr the DCCT/EDIC Research Group. DCCT and EDIC studies in type 1 diabetes: lessons for diabetic neuropathy regarding metabolic memory and natural history. Curr Diab rep 2010; 10: 276-82.

43. Colagiuri S. Optimal management of type 2 diabetes: the evidence. Diab Obes Metab 2012; 14 (suppl. 1): 3-8. DOI: $10.1111 / j .1463-1326.2011 .01506 . x$ 